\title{
A primary current distribution model of a novel micro-electroporation channel configuration
}

\author{
Gregory D. Troszak • Boris Rubinsky
}

Published online: 8 June 2010

(C) The Author(s) 2010. This article is published with open access at Springerlink.com

\begin{abstract}
Traditional macro and micro-electroporation devices utilize facing electrodes, which generate electric fields inversely proportional to their separation distance. Although the separation distances in micro-electroporation devices are significantly smaller than those in macro-electroporation devices, they are limited by cell size. Because of this, significant potential differences are required to induce electroporation. These potential differences are often large enough to cause water electrolysis, resulting in electrode depletion and bubble formation, both of which adversely affect the electroporation process. Here, we present a theoretical study of a novel micro-electroporation channel composed of an electrolyte flowing over a series of adjacent electrodes separated by infinitesimally small insulators. Application of a small, non-electrolysis inducing potential difference between the adjacent electrodes results in radiallyvarying electric fields that emanate from these insulators, causing cells flowing through the channel to experience a pulsed electric field. This eliminates the need for a pulse generator, making a minimal power source (such as a battery) the only electrical equipment that is needed. A non-dimensional primary current distribution model of the novel micro-electroporation channel shows that decreasing the channel height results in an exponential increase in the
\end{abstract}

G. D. Troszak $(\bowtie)$

Department of Mechanical Engineering,

University of California - Berkeley,

6124 Etcheverry Hall,

Berkeley, CA 94720-1740, USA

e-mail: gdtroszak@berkeley.edu

B. Rubinsky

Department of Mechanical Engineering and Bioengineering,

University of California - Berkeley,

Berkeley, CA 94720, USA

e-mail: rubinsky@me.berkeley.edu electric field magnitude, and that cells experience exponentially greater electric field magnitudes the closer they are to the channel walls. Finally, dimensional primary current distribution models of two potential applications, water sterilization and cell transfection, demonstrate the practical feasibility of the novel micro-electroporation channel.

Keywords Electroporation · Micro-electroporation . Primary current distribution model $\cdot$ Sterilization . Transfection

\section{Nomenclature}

$\phi \quad$ electric potential (V)

$\phi_{a} \quad$ electric potential at anode (V)

$\phi_{c} \quad$ electric potential at cathode $(\mathrm{V})$

$\phi_{\text {diff }}$ electric potential difference between electrodes (V)

$L \quad$ active electrode length $(\mu \mathrm{m})$

$H$ half of the micro-electroporation channel height $(\mu \mathrm{m})$

$r \quad$ cell radius $(\mu \mathrm{m})$

$\Phi \quad$ dimensionless electric potential (-)

$\Phi_{a} \quad$ dimensionless electric potential at anode (-)

$\Phi_{c} \quad$ dimensionless electric potential at cathode (-)

$X \quad$ dimensionless $\mathrm{x}$

$Y$ dimensionless y

A micro-electroporation channel aspect ratio (-)

$R \quad$ relative cell radius (-)

E dimensionless electric field (-)

\section{Introduction}

Electroporation is the permeabilization of the cell membrane lipid bilayer due to a pulsed electric field (Weaver 
and Chizmadzhev 1996). Although the physical mechanism that causes electroporation is not fully understood, it is believed that pulsed electric fields significantly increase the potential difference at the cell membrane, resulting in the formation of transient or permanent pores (Sugar et al. 1987; Wong and Neumann 1982). The extent of pore formation primarily depends on the strength and duration of the pulsed electric field, causing membrane permeabilization to be reversible or irreversible (Ho and Mittal 1996). Reversible electroporation is commonly used to transfer macro-molecules such as proteins (Ho et al. 1997), DNA (Prasanna and Panda 1997), and drugs (Tsong and Kinosita 1985) into cells, while the destructive nature of irreversible electroporation makes it suitable for pasteurization or sterilization (Knorr et al. 2001; Mastwijk and Bartels 2004; Wouters et al. 1999).

In a typical electroporation procedure, a suspension of cells is placed between a pair of electrodes and a pulsed electric field is applied. This procedure is capable of treating large quantities of cells. However, because of the large number of cells, electroporation parameters have to be determined based on the average properties of the cell population. As a result, the extent of permeabilization varies throughout the treated cells (Andreason 1993). This undesirable outcome can be remedied by performing electroporation on individual cells, termed single cell micro-electroporation.

The primary advantage of micro-electroporation is the ability to easily handle and manipulate individual cells, making it possible to control the extent of membrane permeabilization through real-time monitoring of pore formation (Fox et al. 2006). For instance, our group developed a micro-electroporation chip that uses a pressure difference to trap individual suspended cells in a micro-hole located between facing electrodes (Huang and Rubinsky 1999). When the micro-hole is open, a voltage pulse between the electrodes produces a measurable current. Conversely, when a cell becomes trapped in the microhole it acts as an electrical insulator between the electrodes, impeding current flow. This provides verification that a cell is trapped. However, if voltage pulses are sufficient to induce electroporation, pores in the cell membrane provide a path for current flow. By measuring this current flow, it is possible to monitor the extent of membrane permeabilization, enabling control of electroporation.

While micro-electroporation devices enable the control of membrane permeabilization, generating high-strength electric fields is a challenge. Most macro and micro-electroporation devices have facing electrodes (Fox et al. 2006). Because of this, the electric field generated between the electrodes is inversely proportional to their separation distance. Although the separation distances in micro-electroporation devices are significantly smaller than those in typical electroporation devices, they are limited by cell size. Since most cells have sizes on the order of $10 \mu \mathrm{ms}$, significant potential differences are required to induce electroporation (Fox et al. 2006). These potential differences are often large enough to cause water electrolysis, resulting in electrode depletion and bubble formation, both of which adversely affect the electroporation process (Fox et al. 2006).

To address this problem, we have conceived a microelectroporation configuration allowing for the unprecedented miniaturization of micro-electroporation devices, which enables the generation of a high-strength electric field with a small, non-electrolysis inducing potential difference. This configuration is composed of an electrolyte flowing over two adjacent electrodes separated by an infinitesimally small insulator. Application of a small potential difference between the adjacent electrodes results in a radially-varying electric field emanating from the infinitesimally small insulator (Fig. 1). This electric field can be used to electroporate cells suspended in the electrolyte. Mirroring the configuration and placing it in series forms a microelectroporation channel with multiple electric fields (Figs. 2 (a) and 3). Cells flowing through this channel will experience a pulsed electric field. The magnitude of this electric field can be adjusted by altering the height of the channel. Furthermore, adjusting the electrolyte flow rate alters the duration of the electric field experienced by cells suspended in the electrolyte.

In this paper we present a theoretical study that investigates the effect of channel geometry and cell size on the electric field in the flowing electrolyte. Additionally, we demonstrate two potential uses of the micro-electroporation channel: water sterilization and cell transfection.

\section{Methods}

A two-dimensional, steady-state, primary current distribution model was developed to understand the effect of micro-electroporation channel geometry and cell size on the electric field in the flowing electrolyte (Fig. 2). Primary current distribution models neglect surface and concentra-

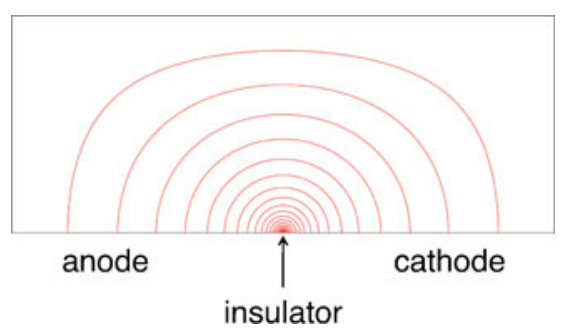

Fig. 1 Electric field streamlines in a micro-electroporation configuration with adjacent electrodes separated by an infinitesimally small insulator. A radially-varying electric field is present 
Fig. 2 (a) Schematic of the micro-electroporation channel configuration. (b) Model domain in the absence of a cell. (c) Model domain in the presence of a cell

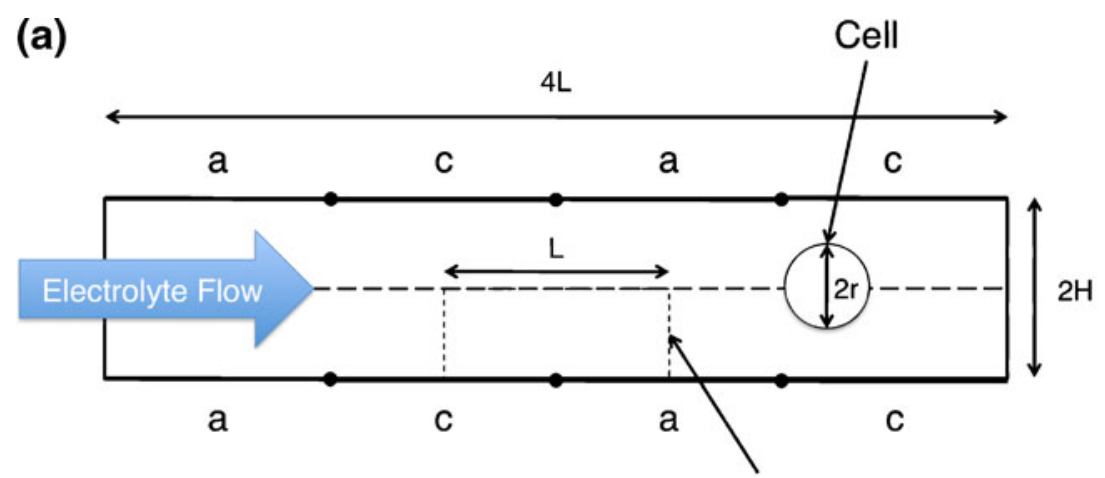

Model Domain

(b)

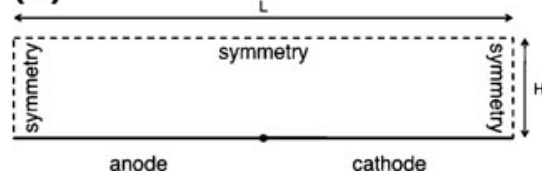

(c)

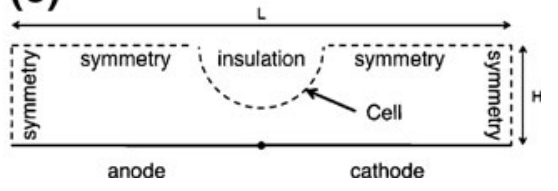

tion losses at the electrode surfaces, only taking into account electric field effects from ohmic losses in the electrolyte. Therefore, primary current distribution models are governed by the Laplace equation:

$\nabla^{2} \phi=0$

where $\phi$ is the electric potential (Prentice 1991). Furthermore, electrode surfaces are assumed to be at a constant potential, making the boundary conditions at the adjacent electrode surfaces:

$\phi_{a}=\phi_{\text {diff }}$ for $\{0<x \leq L / 2 \quad y=0\}$

$\phi_{c}=0$ for $\{L / 2<x \leq L \quad y=0\}$

where $\phi_{a}$ and $\phi_{c}$ are the potentials at the anode and cathode, respectively, $\phi_{\text {diff }}$ is the potential difference between them, and $L$ is the active electrode length. The remaining symmetry boundaries are governed by:

$\nabla \phi=0$ for $\left\{\begin{array}{cc}x=0 & 0<y \leq H \\ x=L & 0<y \leq H \\ 0<x \leq L & y=H\end{array}\right\}$

where $H$ is half of the height of the micro-electroporation channel. Due to the insulating properties of cell mem-
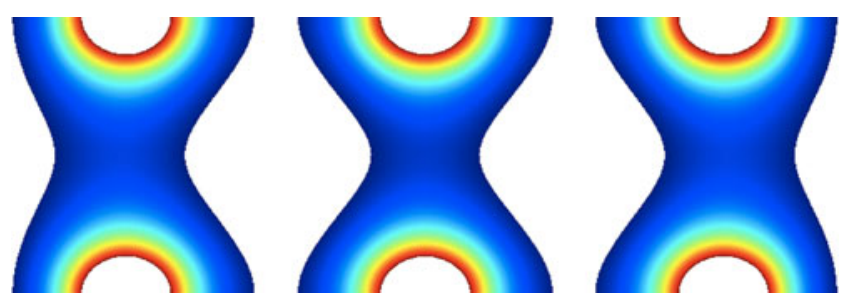

Fig. 3 Radially-varying electric fields generated in the microelectroporation channel branes, cells flowing through the micro-electroporation channel are modeled as electrically insulating boundaries, which are identical to symmetry boundaries.

2.1 Non-dimensionalization of the primary current distribution model

The primary current distribution model was nondimensionalized to analyze the effect of microelectroporation channel geometry and cell size on electric fields in the electrolyte. The Laplace equation in twodimensional Cartesian coordinates is:

$\frac{\partial^{2} \phi}{\partial x^{2}}+\frac{\partial^{2} \phi}{\partial y^{2}}=0$

Substituting the non-dimensional variables:

$\Phi=\phi / \phi_{\text {diff }}$

$X=x / L$

$Y=y / H$

into the Laplace equation yields a non-dimensional form:

$\frac{\partial^{2} \Phi}{\partial X^{2}}+\left(\frac{L}{H}\right)^{2} \frac{\partial^{2} \Phi}{\partial Y^{2}}=0$

Defining the non-dimensional geometry parameter (channel aspect ratio):

$A=\frac{H}{L}$ 
the non-dimensional Laplace equation becomes:

$\frac{\partial^{2} \Phi}{\partial X^{2}}+\frac{1}{A^{2}} \frac{\partial^{2} \Phi}{\partial Y^{2}}=0$

Substitution of the non-dimensional variables into the boundary conditions yields:

$\Phi_{a}=1$ for $\{0<X \leq 0.5 \quad Y=0\}$

$\Phi_{c}=0$ for $\{0.5<X \leq 1 \quad Y=0\}$

$\nabla \Phi=0$ for $\left\{\begin{array}{cc}X=0 & 0<Y \leq 1 \\ X=1 & 0<Y \leq 1 \\ 0<X \leq 1 & Y=1\end{array}\right\}$

Finally, for a spherical cell, the non-dimensional cell radius (relative cell radius) is defined as:

$R=\frac{r}{H}$

where $r$ is the cell radius.

\subsection{Solution of the primary current distribution model}

The non-dimensional primary current distribution model is characterized by the channel aspect ratio $(A)$ and the relative cell radius $(R)$. A parametric study was performed by varying these parameters in a series of models. In each model, the non-dimensional potential distribution was solved for using the finite element analysis software COMSOL Multiphysics 3.5a. A non-dimensional electric field, defined as:

$\mathrm{E}=\nabla \Phi$

was calculated using the non-dimensional potential distribution.

Cells were initially excluded from the models to validate the finite element solution and to better understand how micro-electroporation channel geometry affects the electric field in the electrolyte. These models are only characterized by the channel aspect ratio and have a simplified geometry. This simple geometry, along with the homogenous nature of the non-dimensional Laplace equation and three symmetry boundaries enabled an analytical solution using the separation of variables method. The analytical solution was used to verify the results of the finite element solution. Once the finite element solution was verified, cells were included in the models.

\section{Results}

3.1 Primary current distribution finite element model verification

The non-dimensional primary current distribution finite element model was verified with an analytical solution. Correlation coefficients between the non-dimensional potential distributions of the two solutions were computed in MATLAB (R2007a version 7.4) for values of channel aspect ratio $(A)$ between 0.1 and 1 . The correlation coefficients were 1 for all values of channel aspect ratio, indicating that the finite element and analytical solutions are identical.

3.2 Non-dimensional primary current distribution model results without cells

In the absence of cells, the models are only characterized by the channel aspect ratio $(A)$. As the channel aspect ratio decreases, the magnitude of the non-dimensional electric field increases exponentially in the center of the microelectroporation channel (Fig. 4). Furthermore, highmagnitude non-dimensional electric field contours are more focused and span the height of the channel for small channel aspect ratios (Fig. 5).

3.3 Non-dimensional primary current distribution model results with cells

The electric field in the electrolyte is also affected by the presence of cells. Due to the insulating properties of the cell membrane, electric field contours are compacted (Fig. 6), causing cells to experience exponentially greater electric field magnitudes as the relative cell radius increases $(R)$ (Fig. 7).

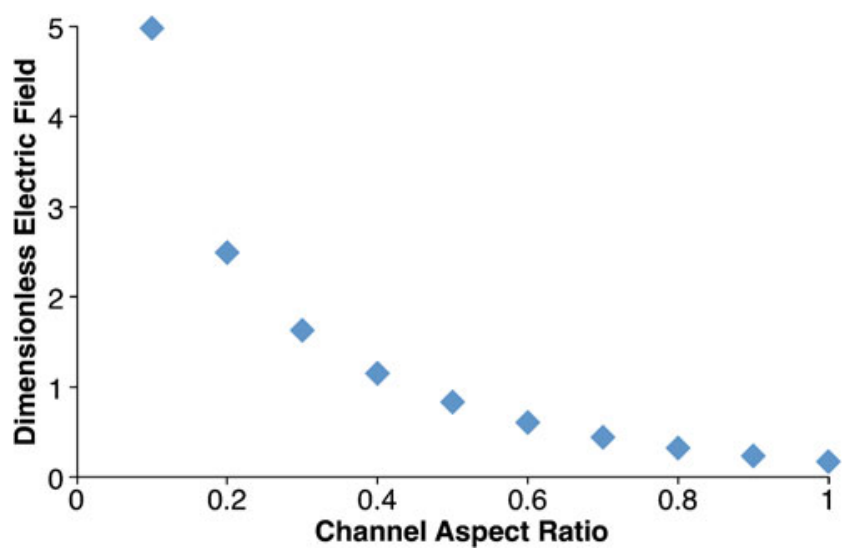

Fig. 4 In the absence of a cell, the dimensionless electric field at $X=$ $0.5, Y=1$ exponentially increases as $A$ decreases. Therefore, larger electric field magnitudes are present in micro-electroporation channels with smaller heights 
Fig. 5 Large dimensionless electric field contours are more focused and span the entire height of the micro-

electroporation channel for small values of $A$
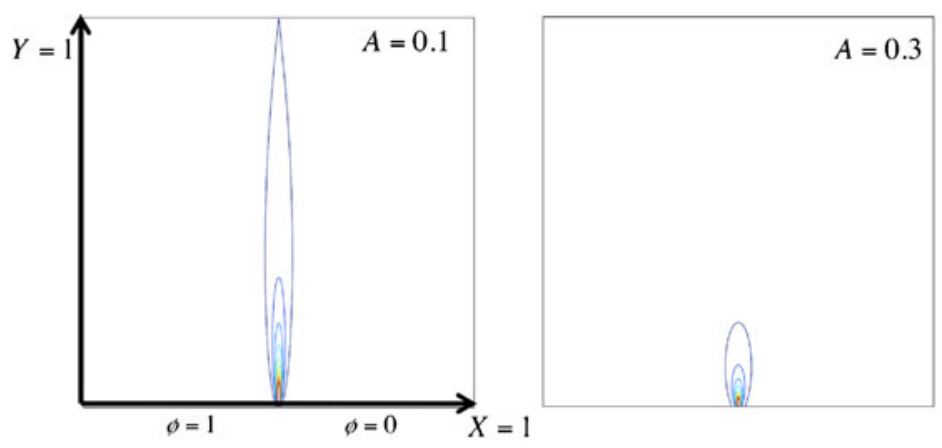

50
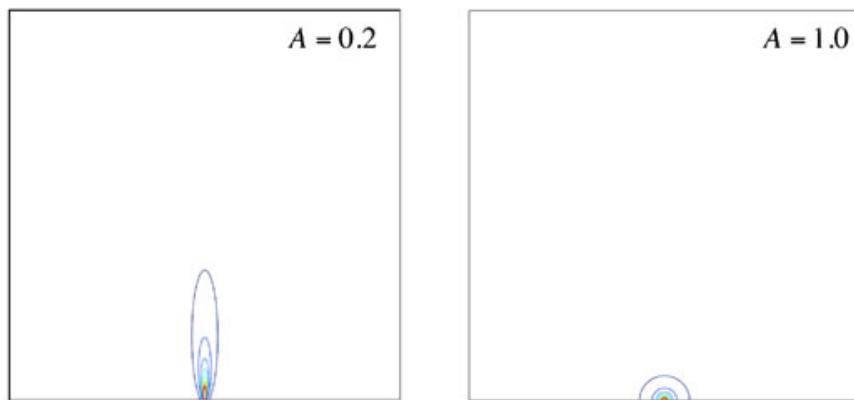

\section{Discussion}

These results show that adjusting micro-electroporation channel height is a way to control the range of electric field magnitudes in the flowing electrolyte without increasing the potential difference between the electrodes. Additionally, models with cells indicate that the closer cells are to the channel walls, the higher electric field magnitudes they will experience.

Although not explored in this paper, it should be noted that changing the length of the insulator separating the adjacent electrodes would affect the electric field in the electrolyte. More specifically, electric field magnitudes throughout the electrolyte would decrease by increasing the length of the insulator.

Traditional macro and micro-electroporation have deficiencies that are addressed by the novel microelectroporation channel. Due to the large quantities of cells treated in macro-electroporation, the extent of cell permeabilization varies throughout the population. While microelectroporation addresses this issue, it typically results in lower throughput. The focused electric fields in the novel
Fig. 6 In the presence of a cell, dimensionless electric field contours are compacted due to the insulating cell membrane
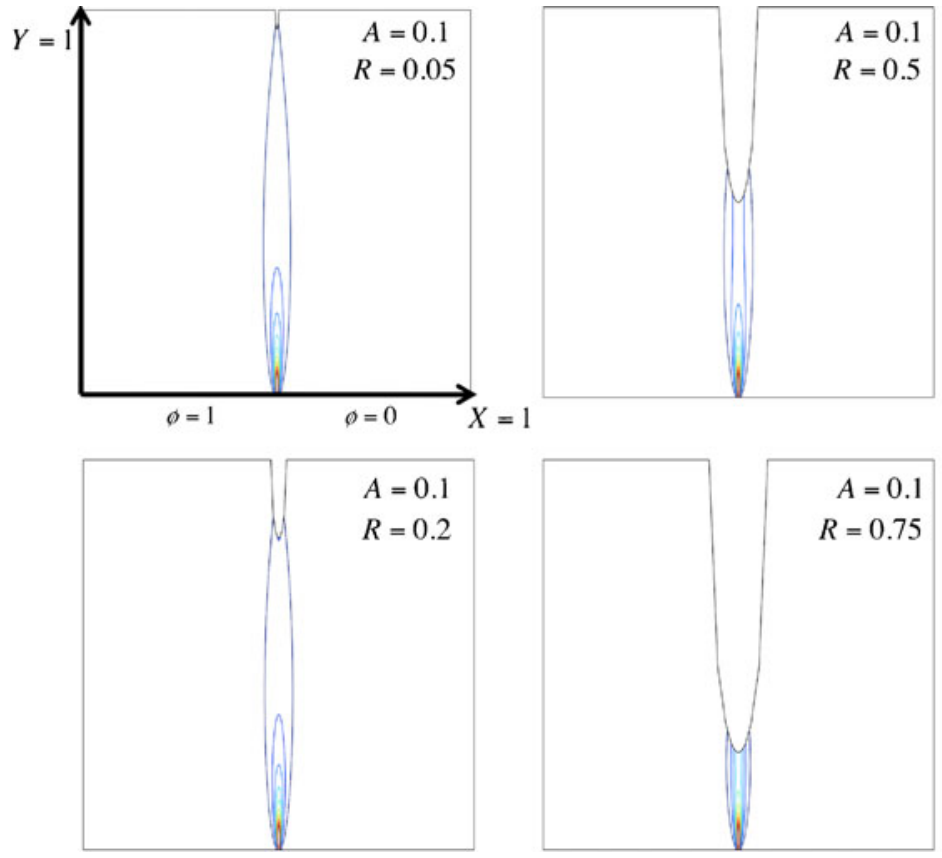

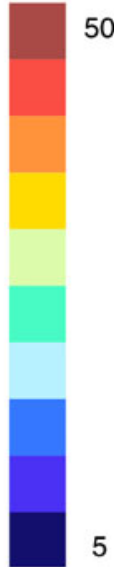




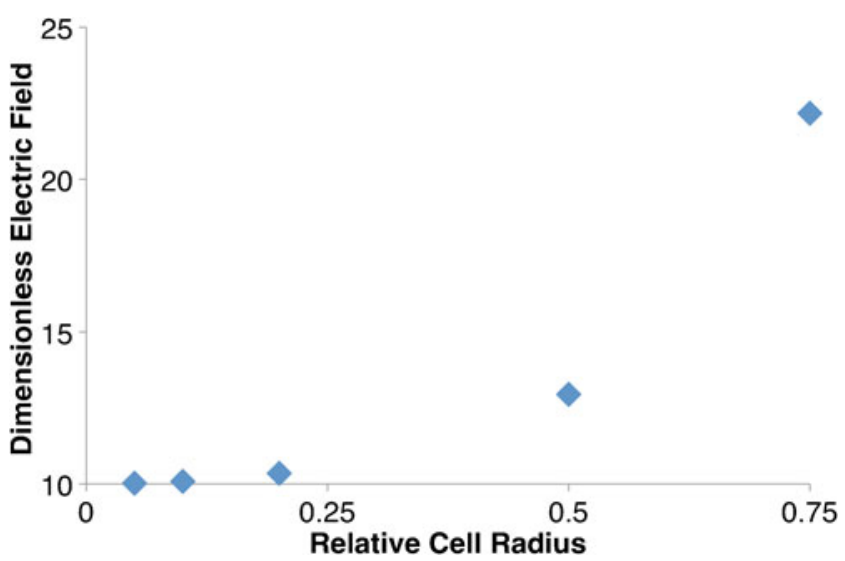

Fig. 7 Cells experience exponentially greater dimensionless electric field magnitudes as $R$ increases for $A=0.1$. Therefore, cells close to the micro-electroporation channel wall will experience larger electric field magnitudes

micro-electroporation channel, which can be modified with channel geometry, offer better control over cell permeabilization than macro-electroporation devices. Additionally, the flow-through nature of the channel makes it suitable for treating large quantities of cells.

Another deficiency addressed by the novel microelectroporation channel is the need for large, electrolysisinducing potential differences in traditional macro and micro-electroporation devices. Most macro and microelectroporation devices have facing electrodes, which results in a uniform electric field that is inversely proportional to their separation distance. Although the separation distances in micro-electroporation devices are significantly smaller than those in typical electroporation devices, they are limited by cell size. Because of this, large, electrolysis-inducing potential differences are required to generate a desired electric field. The novel micro-electroporation channel contains a series of adjacent electrodes separated by infinitesimally small insulators. Application of a small, non-electrolysis-inducing potential difference results in a series of radially-varying electric fields that emanate from the infinitesimally small insulators. Because of this, only a small power source (such as a battery) is required. Reducing the electrical equipment required makes electroporation feasible for a wider range of applications.

\section{Potential applications and conclusions}

\subsection{Potential applications}

The non-dimensional models show that cells of assorted sizes can experience various electric field magnitudes by adjusting the micro-electroporation channel height. Furthermore, the electrolyte flow rate can be used to control exposure time. These parameters enable a great deal of control over the extent of cell permeabilization without the need for complicated electrical equipment, making this concept useful for a number of potential applications including water sterilization and cell transfection.

\subsubsection{Water sterilization}

Contaminated water can cause numerous diseases including diarrhea, which accounts for $4 \%$ of all deaths worldwide (2.2 million). Most of these deaths occur among children under the age of five and represent approximately $15 \%$ of all child deaths under this age in developing nations. It is estimated that sanitation and hygiene intervention could reduce diarrheal infection by one-quarter to one-third; however, this requires access to sterile water, which can be scarce, particularly in rural areas of developing nations (World Health Organization 2000).

Enterotoxigenic Escherichia coli (ETEC, a type of E. coli) is a $2 \mu \mathrm{m}$ long, $0.5 \mu \mathrm{m}$ diameter, rod-shaped fecal coliform, and is the leading bacterial cause of diarrhea in developing nations. Currently, vaccination is the most effective method of preventing diarrhea caused by ETEC. However, vaccines are not available in developing nations where ETEC is endemic (World Health Organization 2009).

It is possible to destroy ETEC with irreversible electroporation using the concept presented in this paper. The results of a dimensional form of the primary current distribution model show that ETEC cells in water flowing through the center of a $0.6 \mu \mathrm{m}$ high micro-electroporation channel with a $0.1 \mathrm{~V}$ potential difference between adjacent electrodes experience electric field magnitudes between 1000 and $10000 \mathrm{~V} / \mathrm{cm}$, inducing irreversible electroporation (Fig. 8) (Sale and Hamilton 1967). It should be noted that this is a conservative estimate, since cells flowing through the center of the channel will experience relatively low strength electric fields compared to cells flowing closer to the electrodes.

\subsubsection{Cell transfection}

Cell transfection is the process of introducing large molecules, primarily nucleic acids and proteins, into cells. These large molecules typically enter cells through transient pores created in the cell membrane by chemical and physical methods, such as electroporation. However, due to the bulk nature of the process, it is difficult to determine the optimal electroporation parameters for high transfection efficiency and minimal cell death (Andreason 1993). Traditional micro-electroporation could remedy this problem; however, traditional micro-electroporation is not appropriate for treating large quantities of cells.

In contrast, the flow-through nature of the microelectroporation channel presented in this paper makes it ideal 


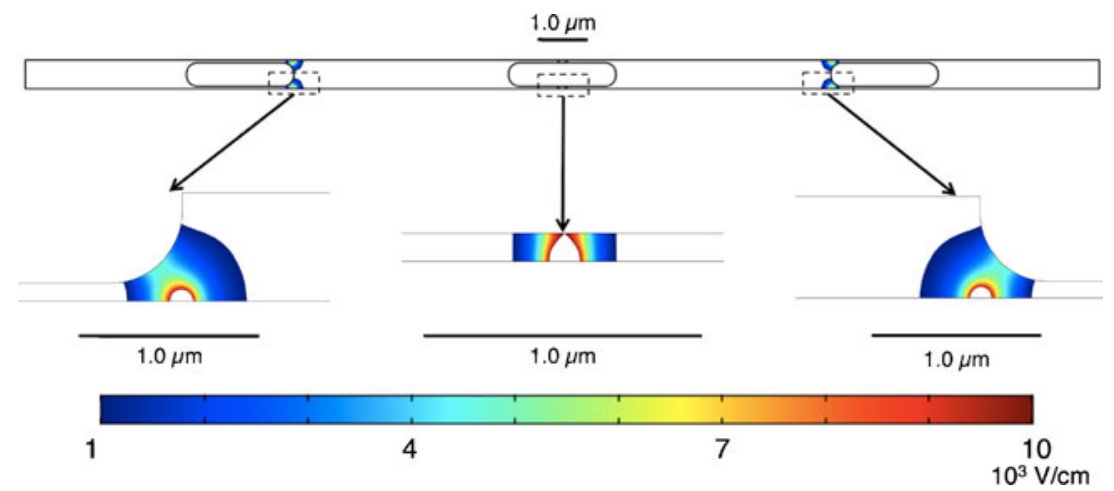

Fig. 8 Enterotoxigenic Escherichia coli (ETEC, a type of E. coli) cells flowing through a $0.6 \mu \mathrm{m}$ high micro-electroporation channel with a $0.1 \mathrm{~V}$ potential between the electrodes. Electric field magnitudes between 1 and $10 \mathrm{kV} / \mathrm{cm}$ are shown for clarity. ETEC

for treating many cells while maintaining control of the electric fields they experience. Yeast is a $4 \mu \mathrm{m}$ diameter cell widely used in genetic research because it is a simple cell that serves as a representative eukaryotic model (Alberts et al. 2007). A dimensional form of the primary current distribution model shows that yeast cells flowing through a $4.2 \mu \mathrm{m}$ high channel with a potential of $0.1 \mathrm{~V}$ between the electrodes experience reversible electroporation inducing electric field magnitudes, creating the transient pores needed for cell transfection (Fig. 9) (Sale and Hamilton 1967). By stacking multiple micro-electroporation channels atop one another, it would be possible to increase throughput while maintaining consistent electric fields.

\subsection{Conclusions}

We have conceptualized a novel micro-electroporation configuration that allows for the unprecedented miniaturization of micro-electroporation devices, enabling the generation of a cells experience irreversible electroporation inducing electric fields $(\sim 10 \mathrm{kV} / \mathrm{cm})$ as they flow over the infinitesimally small insulators. The micro-electroporation channel could be used for water sterilization in developing nations

high-strength electric field with a small, non-electrolysis inducing potential difference. This configuration is composed of an electrolyte flowing over two adjacent electrodes separated by an infinitesimally small insulator. Application of a small potential difference between the adjacent electrodes results in a radially-varying electric field emanating from the infinitesimally small insulator. This electric field can be used to electroporate cells suspended in the electrolyte. Mirroring the configuration and placing it in series forms a microelectroporation channel. Cells flowing through this channel will experience a pulsed electric field whose magnitude can be adjusted by altering the height of the channel. Furthermore, adjusting the electrolyte flow rate alters the duration of the electric field experienced by cells suspended in the electrolyte.

A two-dimensional, steady-state, primary current distribution model was developed to understand the effect of microelectroporation channel geometry and cell size on the electric field in the flowing electrolyte. In the absence of cells, decreasing the micro-electroporation channel height results in
Fig. 9 Yeast cells flowing through a $4.2 \mu \mathrm{m}$ high microelectroporation channel with a $0.1 \mathrm{~V}$ potential between the electrodes. Electric field magnitudes between 1 and $3 \mathrm{kV} / \mathrm{cm}$ are shown for clarity. Yeast cells experience reversible electroporation inducing electric fields $(1-3 \mathrm{kV} / \mathrm{cm})$ as they flow past the infinitesimally small insulators. The micro-electroporation channel could be a cheaper, more efficient method for cell transfection

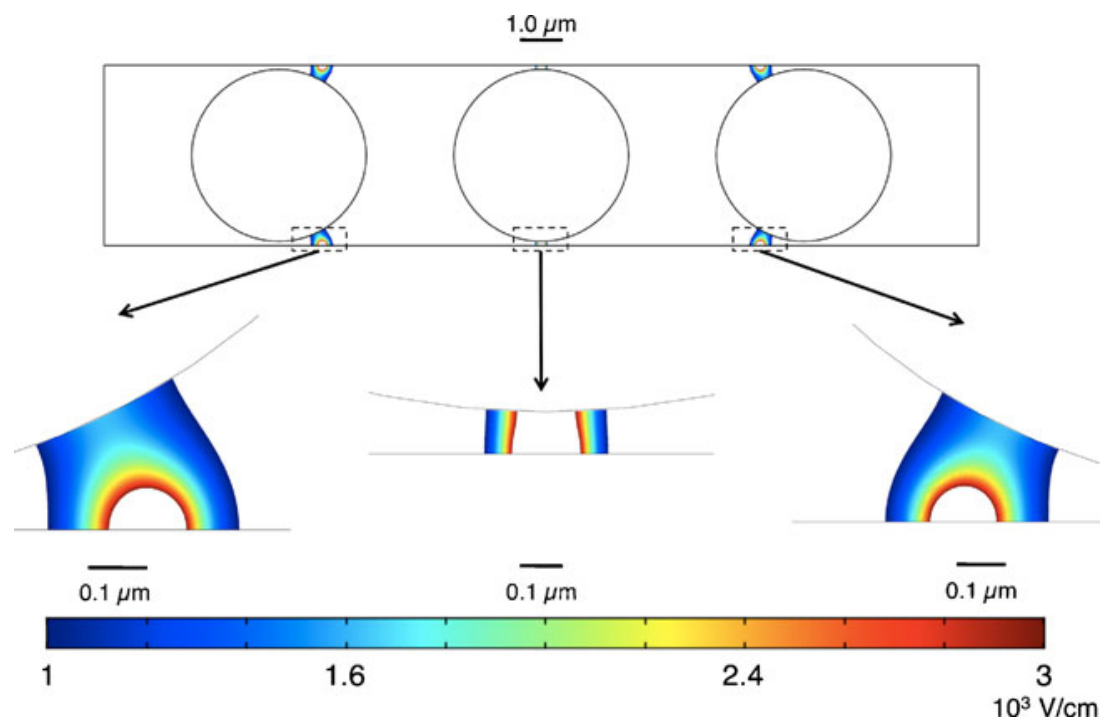


an exponential increase in the electric field magnitude in the center of the channel. Additionally, cells experience exponentially greater electric field magnitudes the closer they are to the micro-electroporation channel walls.

The novel micro-electroporation channel differs from traditional macro and micro-electroporation devices in several ways. In electroporation devices with facing electrodes, a cell's proximity has no bearing on the electric field magnitude it will experience. Conversely, in the micro-electroporation channel, the electric field magnitude experienced by a cell is dictated by the gap between the cell and the channel wall. Because of this, cell size does not affect the potential difference required to achieve a desired electric field.

Another difference between the novel micro-electroporation channel and traditional macro and micro-electroporation devices is that less electrical equipment is required. Traditional macro and micro-electrporation devices require a pulse generator and power supply. However, in the novel microelectroporation channel, the need for a pulse generator is eliminated since it contains a series of adjacent electrodes. Furthermore, since the novel micro-electroporation channel only requires a small potential difference, a minimal power source (such as a battery) is needed.

The simplicity of electroporation makes it a powerful technology. The novel micro-electroporation channel increases the accessibility of electroporation, making its use feasible for a wide range of non-traditional applications.

Acknowledgements This research was supported by funds provided by the Arnold and Barbara Silverman Distinguished Professorship in Bioengineering.

Open Access This article is distributed under the terms of the Creative Commons Attribution Noncommercial License which per- mits any noncommercial use, distribution, and reproduction in any medium, provided the original author(s) and source are credited.

\section{References}

B. Alberts, A. Johnson, J. Lewis, M. Raff, K. Roberts, P. Waiter, Molecular Biology of the Cell, 4th edn. (Garland, New York, 2007)

G.L. Andreason, Tiss. J. Cult. Meth. 15, 56-62 (1993)

Diarrhoeal diseases. (World Health Organization, 2009), http://www. who.int/vaccine_research/diseases/diarrhoeal/en/print.html. Accessed 12 April 2010

M.B. Fox, D.C. Esveld, A. Valero, R. Luttge, H.C. Mastwijk, P.V. Bartels, A. van der Berg, R.M. Boom, Anal. Bioanal. Chem. 385 (3), 474-485 (2006)

Global water supply and sanitation assessment report. (World Health Organization/United Nations Children's Fund, Water Supply and Sanitation Collaborative Council, 2000), http:/www.who.int/ docstore/water_sanitation_health/Globassessment/GlobalTOC. htm. Accessed 12 April 2010

S.Y. Ho, G.S. Mittal, Crit. Rev. Biotechnol. 16(4), 349-362 (1996)

S.Y. Ho, G.S. Mitall, J.D. Cross, J. Food Eng. 31(1), 69-84 (1997)

Y. Huang, B. Rubinsky, Biomed. Microdevices 2(2), 145-150 (1999)

D. Knorr, A. Angersbach, M.N. Eshtiaghi, V. Heinz, D. Lee, Trends Food Sci. Tech. 12(3-4), 129-135 (2001)

H.C. Mastwijk, P.V. Bartels, Int. Rev. Food Sci. Technol. 3, 106-108 (2004)

G.L. Prasanna, T. Panda, Bioprocess Biosyst. Eng. 16(5), 261-264 (1997)

G. Prentice, Electrochemical Engineering Principles, 1st edn. (Prentice, Upper Saddle River, 1991)

A.J.H. Sale, W.A. Hamilton, Biochim. Biophys. 148(3), 781-788 (1967)

I.P. Sugar, W. Förster, E. Neumann, Biophys. Chem. 26(3), 321-335 (1987)

T.Y. Tsong, K. Kinosita, Bibl. Haematol. 51, 108-114 (1985)

J.C. Weaver, Y.A. Chizmadzhev, Bioelectrochem. Bioenerg. 41(2), 135-160 (1996)

T. Wong, E. Neumann, Biochem. Biophys. Res. Commun. 107(2), 584-587 (1982)

P.C. Wouters, N. Dutreux, J.P.P.M. Smelt, H.L.M. Lelieveld, Appl. Environ. Microbiol. 65(12), 5364-5371 (1999) 